\title{
Sobre Martelos, Pregos e Nossa Caixa de Ferramentas, que Sempre Aumenta...
} Ver artigo relacionado
na página 178

\author{
John F. Canales ${ }^{1}$, James J. Ferguson ${ }^{1}$
}

\author{
"Quando você está usando um martelo, tudo parece \\ prego."
}

E sa expressão da sabedoria popular é algo que nós, médicos, deveríamos levar em consideração. Dentro do variado arsenal terapêutico de que dispomos, algumas das armas são mais favorecidas que outras, não necessariamente por serem melhores, mas porque temos mais experiência ou nos sentimos mais seguros ao utilizá-las. Preferimos utilizar as ferramentas (fármacos, dispositivos) com as quais temos maior familiaridade, e quando uma delas funciona bem (ou assim acreditamos) tendemos a usá-la com maior freqüência. Em muitos aspectos, a forma com que utilizamos a terapia antitrombótica na intervenção coronária é um exemplo perfeito de que procuramos não só preservar aquilo que nos dá mais segurança, mas, também, encontrar maneiras de explorar outras opções terapêuticas, sem, no entanto, nos desviarmos muito de nossa "zona de segurança".

A terapia antitrombótica é atualmente recomendada pelo American College of Cardiology, pela American Heart Association e pela Sociedade Européia de Cardiologia para uso durante a intervenção coronária percutânea (ICP), com a finalidade de evitar a formação de trombo no local do dano vascular infligido pelo instrumental de dilatação percutâneo, dentro de cateteres e nos guias $^{1,2}$. Historicamente, a heparina não-fracionada (HNF) tem sido o sustentáculo da terapêutica anticoagulante durante a ICP; no entanto, em decorrência das inúmeras limitações clínicas e bioquímicas, novas terapias antitrombóticas, incluindo heparinas de baixo peso molecular (HBPM), inibidores diretos da trombina e inibidores diretos e indiretos do fator Xa, passaram a fazer parte de um arsenal terapêutico cada vez maior.

As HBPM, tais como a enoxaparina, surgiram como uma alternativa atraente para a HNF na anticoagulação sistêmica, pelas inúmeras limitações biológicas e farmacocinéticas apresentadas pela HNF. A inativação da trombina pela HNF depende da ligação da antitrombina e da trombina e é dependente do comprimento da cadeia molecular. No entanto, a maior parte dos efeitos colaterais indesejados da HNF depende do comprimento da cadeia (e de sua carga), incluindo significante ligação com as proteínas (levando a biodisponibilidade inconsistente e graus variáveis e pouco confiáveis da anticoagulação), ativação plaquetária significativa, risco de trombocitopenia induzida pela heparina e incapacidade para bloquear a trombina ligada ao coágulo ${ }^{3-5}$.

Embora a HBPM (administrada por via subcutânea) já esteja estabelecida como parte integrante do tratamento medicamentoso moderno da síndrome coronária aguda, o uso da HBPM como anticoagulante alternativo para uso no procedimento durante a ICP eletiva (ou urgente) está bem menos estabelecido. Uma série de estudos tem analisado o uso da enoxaparina por via intravenosa (IV) como alternativa para HNF na ICP eletiva $^{6-9}$. Nesses estudos relativamente pequenos, a HBPM, administrada por via IV e não por via subcutânea, em cenário de ICP eletiva, foi tão eficaz quanto a HNF, com risco semelhante de hemorragia. O STEEPLE ${ }^{10}$ é o estudo prospectivo em grande escala mais recente comparando a HNF à enoxaparina IV na ICP eletiva. Nesse estudo, 3.528 pacientes foram randomizados para enoxaparina IV $(0,5 \mathrm{mg} / \mathrm{kg}$ ou 0,75 mg/kg) ou HNF IV (50-70 U/kg ou 70-100 U/kg), com ou sem a utilização de antagonista da glicoproteína Ilb/Illa. O objetivo primário, o sangramento maior ou menor em 48 horas não relacionado à cirurgia de revascularização miocárdica, ocorreu em 6\%, 6,6\% e $8,7 \%$ dos grupos enoxaparina IV $0,5 \mathrm{mg} / \mathrm{kg}$, enoxaparina IV $0,75 \mathrm{mg} / \mathrm{kg}$ e HNF IV $(\mathrm{p}=0,014$ para enoxaparina

\footnotetext{
1 Texas Heart Institute at St. Luke's Episcopal Hospital, Houston, Texas, Estados Unidos.

Correspondência: John F. Canales - Texas Heart Institute at St. Luke's Episcopal Hospital - 6720 Bertner, M/C 1-133 - Houston, TX, USA - 77030 E-mail: jcanales1@sleh.com

Recebido em: 27/5/2008 • Aceito em: 30/5/2008
} 
$0,5 \mathrm{mg} / \mathrm{kg}$ versus $\mathrm{HNF}$, e $\mathrm{p}=0,052$ para enoxaparina $0,75 \mathrm{mg} / \mathrm{kg}$ versus HNF). Não houve diferença estatisticamente significante na morte, no infarto agudo do miocárdio (IAM) não-fatal ou na revascularização de urgência do vaso-alvo em 30 dias.

Pela análise multivariada, a designação para o grupo enoxaparina foi preditor independente da redução de sangramentos maiores e menores. Apesar dos dados sobre segurança da enoxaparina IV fornecidos pelo STEEPLE ${ }^{10}$, uma série de questões ainda continua sem resposta, incluindo os resultados a longo prazo, a dosagem ideal, e a extensão e o significado clínico da inibição do fator anti-Xa alcançada.

Nesta edição da Revista Brasileira de Cardiologia Invasiva, Centemero et al. ${ }^{11}$ fornecem informações adicionais sobre a segurança e a eficácia da enoxaparina IV na ICP eletiva. Os autores descrevem a experiência de único centro, prospectiva, aberta, com pacientes consecutivos recebendo enoxaparina IV $(0,75 \mathrm{mg} / \mathrm{kg}$; $\mathrm{n}=57)$ na ICP eletiva, em comparação com sua experiência de HNF IV (100 UI/kg; $n=143)$ em pacientes com perfil semelhante. A ICP foi realizada em uso de terapêutica oral com aspirina e tienopiridínico (sem a especificação do uso de inibidores da glicoproteína Ilb/IIla). Os níveis de anti-Xa foram medidos 10 minutos após o bolus IV de enoxaparina e no final do procedimento. Os TCAs e os níveis de anti-Xa do grupo HNF não foram relatados.

Os pacientes foram monitorizados para o objetivo primário de sangramento TIMI maior e menor e eventos cardiovasculares maiores (morte, IAM, acidente vascular cerebral, revascularização de urgência) durante a hospitalização e não foram observadas diferenças significativas relacionadas à freqüência desses eventos nessa população de risco relativamente baixo. Os pacientes foram subseqüentemente acompanhados por três anos após a ICP, tendo sido comparadas as freqüências combinadas de morte, IAM, acidente vascular cerebral e necessidade de nova revascularização. Nesse período, também não foram observadas diferenças entre os grupos nos eventos cardiovasculares maiores. Os níveis de anti-Xa, 10 minutos após o bolus IV, foram de 1,21 $\pm 0,23 \mathrm{UI} / \mathrm{ml}(0,73$ $1,68 \mathrm{Ul} / \mathrm{ml})$ e 1,04 \pm 0,23 Ul/ml (0,56-1,61 Ul/ml) após o procedimento. Não foram realizadas análises para explorar potenciais relações entre níveis de anti-Xa e eventos clínicos, tampouco a precisa caracterização de início e término da atividade anti-Xa.

Vários aspectos deste estudo merecem ser comentados. Este estudo foi um estudo observacional, relativamente pequeno, realizado em único hospital, com uma população de pacientes de complexidade baixa a moderada para ICP eletiva. Poucos detalhes são fornecidos sobre a terapia adjuvante (ou o grau de anticoagulação) no grupo controle. Exceto pela documentação da atividade anti-Xa no grupo da enoxaparina (por si só uma contribuição valiosa), não foram realizadas análises posteriores do grau de anticoagulação com enoxaparina ou HNF ou de sua vinculação com os resultados. Não faltam, no entanto, oportunidades para se aprender ainda mais.

No que diz respeito à segurança da enoxaparina IV $(0,75 \mathrm{mg} / \mathrm{kg})$, não ocorreram sangramentos TIMI maiores e cinco sangramentos TIMI menores ocorreram nos grupos HNF e enoxaparina IV $(3,5 \%$ versus $8,8 \% ; p=0,15)$. Embora sem significância estatística, os eventos hemorrágicos tenderam a ser mais elevados no grupo enoxaparina IV que no grupo HNF, em contraste com os resultados do STEEPLE ${ }^{10}$, que apresentou menores taxas de hemorragia com enoxaparina. Os autores utilizaram controles tratados com HNF, administrada em doses de $100 \mathrm{U} / \mathrm{kg}$, mas não mencionaram a metodologia para a dosagem do tempo de coagulação ativada (TCA) ou relataram o uso suplementar de HNF.

A atividade anti-Xa nesse estudo parece ter sido avaliada com método diferente do utilizado pelos investigadores do STEEPLE ${ }^{10}$, o que inviabiliza as comparações entre os estudos. Ainda não está totalmente definida a "faixa terapêutica" de escolha para a atividade antiXa da HBPM utilizada nos procedimentos. Estudos prévios têm sugerido eventos cardiovasculares reduzidos com níveis anti-Xa $>0,5 \mathrm{Ul} / \mathrm{ml}$, mas esse alvo terapêutico é, em grande parte, oriundo da literatura relacionada a tratamento/profilaxia da trombose venosa profunda, e não pode, nem deve, ser extrapolado para processos arteriais trombóticos, que podem ter fundamentos mecanicistas completamente diferentes. Provavelmente as informações mais valiosas do estudo de Centemero et al. ${ }^{11}$ residem na distribuição das curvas da Figura 1.

Por último, 36\% da população do estudo realizou ICP eletiva na vigência de "quadro clínico instável", sendo $21,5 \%$ descritos como portadores de síndrome coronária aguda sem supradesnivelamento do segmento ST (embora presumivelmente sem alterações dinâmicas do eletrocardiograma ou biomarcadores alterados, como relatado nos critérios de exclusão). Não se sabe se os pacientes com quadros agudos receberam outros anticoagulantes (cross-over ou stacking [= empilhamento, amontoamento]) ou agentes antiplaquetários (glicoproteína IIb/IIla) antes da ICP eletiva. Com base nos resultados do estudo SYNERGY ${ }^{12}$, sabe-se que acrescentar HNF à enoxaparina de maneira não-controlada (stacking therapy), em oposição a uma transição controlada, guiada por protocolo, pode aumentar as complicações hemorrágicas.

No que se refere aos dados de eficácia, Centemero et al. ${ }^{11}$ descrevem os eventos cardiovasculares maiores durante a hospitalização e aos três anos de acompanhamento. Eventos cardiovasculares maiores foram registrados durante a hospitalização, sem mencionar $\mathrm{o}$ período de permanência hospitalar dos pacientes, nem 
em que momento os eventos foram detectados. Não se observaram diferenças estatisticamente significantes entre HNF e enoxaparina durante a hospitalização (nenhum evento clínico foi detectado nesse período). Incidência de creatina quinase fração $M B(C K-M B)>$ 3 vezes o limite normal foi observada em dois casos no grupo enoxaparina, sem pormenores específicos da anatomia ou de eventos associados. Obviamente, com esse pequeno número de pacientes não existe a possibilidade estatística de detectar diferenças com eventos muito pouco freqüentes. Aos três anos de acompanhamento, alguns eventos adicionais ocorreram nos dois grupos, sem diferenças significativas entre eles (35 x 18 no grupo enoxaparina; $p=0,47)$.

Um último ponto digno de nota é o crescente reconhecimento das diferenças na atividade farmacodinâmica da enoxaparina quando administrada por via intravenosa, comparativamente à administração por via subcutânea. Em contraposição à visão clínica geral de que o TCA e o tempo de tromboplastina parcial ativada (TTPa) não são úteis para a monitorização da HBPM, diversos estudos têm demonstrado que, quando administrada por via intravenosa, a HBPM (enoxaparina e dalteparina) altera significativamente tanto $\mathrm{O}$ TCA como o TTPa. No entanto, em estudos de HBPM por via subcutânea, essas alterações não são observadas. Como se explica isso? Esse enigma pode ser explicado pela hipótese de que a HBPM por via subcutânea pode ter atividade absoluta anti-Ila significativamente menor (talvez relacionada à ligação mais ávida das cadeias mais longas de heparina à proteína nos tecidos) e pouco ou nenhum efeito sobre o TCA e o TTPa, enquanto a HBPM IV pode acarretar menos "filtragem" da atividade anti-Ila e, proporcionalmente, maior efeito anti-Ila. Em sua essência, essas duas vias de administração podem se apresentar como equivalentes a dois agentes farmacológicos totalmente diferentes. Dada a importância da inibição da atividade da trombina (efeito anti-Ila) durante a ICP, a melhor compreensão dos efeitos farmacodinâmicos da HBPM IV se faz necessária. Já sabemos que a farmacocinética (início/término da ação) da HBPM IV está muito mais próxima da HNF IV, o que nos leva a crer que suas farmacodinâmicas (efeito Xa/Ila) estejam muito mais próximas entre elas que da HBPM subcutânea. Por ora continua a ser uma hipótese, mas com profundas implicações clínicas.

Em resumo, embora não dê respostas definitivas sobre a segurança e a eficácia da enoxaparina IV na ICP eletiva, o estudo de Centemero et al. ${ }^{11}$ fornece informações valiosas adicionais, especialmente sobre a atividade anti-Xa após bolus de enoxaparina IV. Estudos anteriores, incluindo o STEEPLE ${ }^{10}$, têm demonstrado que a enoxaparina IV pode ser seguramente administrada a pacientes de baixo risco submetidos a ICP eletiva.

O estudo atual corrobora esses achados e nos mostra um acompanhamento a longo prazo, embora com número limitado de pacientes. Mais dados explorando os resultados da intervenção em função do grau de anticoagulação, melhor caracterização da ascensão e da queda da atividade anti-Xa, e maior número de pacientes que permita análise válida dos subgrupos clínicos e da terapêutica antiagregante concomitante serão úteis no futuro.

Estamos avançando além da maneira única de utilizar o martelo. E como nossa caixa de ferramentas está em constante expansão, é preciso lembrar, acima de tudo, de que não é somente com os instrumentos que precisamos nos preocupar, mas com a habilidade e a experiência da pessoa que utiliza esses instrumentos, pois são essas qualidades que fazem a diferença entre um bom operador e outro verdadeiramente magistral. O conceito de "tamanho único", na Cardiologia Intervencionista, não é adequado para todos, e, com o tempo, seremos capazes de perceber as nuances clínicas do melhor emprego das ferramentas de que dispomos, como a enoxaparina IV. Ainda temos muito a aprender.

\section{REFERÊNCIAS BIBLIOGRÁFICAS}

1. Smith SC Jr, Feldman TE, Hirshfeld JW, Jacobs AK, Kern MJ King SB 3 $3^{\text {rd }}$, et al. ACC/AHA/SCAI 2005 Guideline Update for Percutaneous Coronary Interventions: a report of the American College of Cardiology/American Heart Association Task Force on Practice Guidelines (ACC/AHA/SCAI Writing Committee to Update the 2001 Guidelines for Percutaneous Coronary Intervention). J Am Coll Cardiol. 2006;47:1-121.

2. Silber S, Albertsson P, Aviles FF, Camici PG, Colombo A, Hamm C, et al. Guidelines for percutaneous coronary interventions: the Task Force for Percutaneous Coronary Interventions of the European Society of Cardiology. Eur Heart J. 2005;26(8):804-47.

3. Young E, Wells P, Holloway S, Weitz J, Hirsh J. Ex-vivo and in-vitro evidence that low molecular weight heparins exhibit less binding to plasma proteins than unfractionated heparin. Thromb Haemost. 1994;71(3):300-4.

4. Warkentin TE, Levine MN, Hirsh J, Horsewood P, Roberts RS, Gent $M$, et al. Heparin-induced thrombocytopenia in patients treated with low-molecular-weight heparin or unfractionated heparin. N Engl J Med. 1995;332(20):1330-5.

5. Bhandari M, Hirsh J, Weitz JI, Young E, Venner TJ, Shaughnessy SG. The effects of standard and low molecular weight heparin on bone nodule formation in vitro. Thromb Haemost. 1998; 80(3):413-7.

6. Rabah MM, Premmereur J, Graham M, Fareed J, Hoppensteadt DA, Grines LL, et al. Usefulness of intravenous enoxaparin for percutaneous coronary intervention in stable angina pectoris. Am J Cardiol. 1999;84(12):1391-5.

7. KeZreiakes DJ, Grines C, Fry E, Esente P, Hoppensteadt D, Midei $M$, et al. Enoxaparin and abciximab adjunctive pharmacotherapy during percutaneous coronary intervention. J Invasive Cardiol. 2001;13(4):272-8.

8. Bhatt DL, Lee BI, Casterella PJ, Pulsipher $M$, Rogers $M$, Cohen M, et al. Safety of concomitant therapy with eptifibatide and enoxaparin in patients undergoing percutaneous coronary intervention: results of the Coronary Revascularization Using Integrilin and Single bolus Enoxaparin Study. J Am Coll Cardiol. 2003;41(1):20-5. 
9. Choussat R, Montalescot G, Collet JP, Vicaut E, Ankri A, Gallois $V$, et al. A unique, low dose of intravenous enoxaparin in elective percutaneous coronary intervention. J Am Coll Cardiol. 2002;40(11):1943-50.

10. Montalescot G, White HD, Gallo R, Cohen M, Steg PG, Aylward PE, et al.; STEEPLE Investigators. Enoxaparin versus unfractionated heparin in elective percutaneous coronary intervention. N Engl J Med. 2006;355(10):1006-17.

11. Centemero M, Maia F, Sousa AGMR, Feres F, Staico R, Mattos LA, et al. Segurança e efetividade da enoxaparina versus heparina não-fracionada no tratamento de pacientes submetidos a implante eletivo de stents não-farmacológicos: resultados imediatos e tardios. Rev Bras Cardiol Invas. 2008;16(2):178-84.

12. Ferguson JJ, Califf RM, Antman EM, Cohen M, Grines CL, Goodman S, et al.; SYNERGY Trial Investigators. Enoxaparin vs unfractionated heparin in high-risk patients with non-STsegment elevation acute coronary syndromes managed with an intended early invasive strategy: primary results of the SYNERGY randomized trial. JAMA. 2004;292(1):45-54. 